Kanmaz, B., Lappin, D. F., Nile, C. J. and Buduneli, N. (2020) Effects of smoking on non-surgical periodontal therapy in patients with periodontitis stage III or IV, and grade C. Journal of Periodontology, 91(4), pp. 442-453.

There may be differences between this version and the published version. You are advised to consult the publisher's version if you wish to cite from it.

This is the peer reviewed version of the following article:

Kanmaz, B., Lappin, D. F., Nile, C. J. and Buduneli, N. (2020) Effects of smoking on non-surgical periodontal therapy in patients with periodontitis stage III or IV, and grade C. Journal of Periodontology, 91(4), pp. 442-453. (doi: 10.1002/JPER.19-0141)

This article may be used for non-commercial purposes in accordance with Wiley Terms and Conditions for Self-Archiving.

http://eprints.gla.ac.uk/195387/

Deposited on: 30 September 2019

Enlighten - Research publications by members of the University of Glasgow http://eprints.gla.ac.uk/ 


\title{
Effects of Smoking on Non-surgical Periodontal Therapy in Patients with Periodontitis Stage III or IV, and Grade C
}

\author{
Burcu KANMAZ ${ }^{*}$ DDS, PhD \\ David F LAPPIN ${ }^{\dagger}$ BSc PhD \\ Christopher J NILE ${ }^{\dagger}$ BSc PhD \\ Nurcan BUDUNELİ DDS, PhD, Professor
}

${ }^{*}$ Department of Periodontology, School of Dentistry, Ege University, Izmir, Turkey.

${ }^{\dagger}$ Oral Sciences Research Group, School of Medicine, Dentistry and Nursing, Glasgow Dental School, University of Glasgow, UK.

Number of words: 3990 (excluding the abstract and references)

Number of tables: 2

Number of figures: 5

Number of references: 43

Running title: Smoking and non-surgical treatment

One sentence summary: Smoker periodontitis patients exhibit higher detection rates of Gram-negative bacteria before and after non-surgical periodontal therapy.

\section{Corresponding author:}

Dr. Nurcan Buduneli

Department of Periodontology

School of Dentistry

Ege University

35100 Bornova, İzmir / Turkey

Tel: + 902323114556

Fax: + 902323880325

E-mail: nurcan.buduneli@ege.edu.tr 


\section{ABSTRACT}

Aim: To evaluate possible effects of smoking on clinical, biochemical, and microbiological outcomes of non-surgical periodontal treatment in patients with periodontitis stage III or IV.

Material and Method: Conventional quadrant-wise non-surgical periodontal treatment was performed and whole-mouth periodontal measurements were recorded at baseline, 1-, 3-, 6months after completion of treatment. Saliva, gingival crevicular fluid, subgingival plaque, and blood samples were obtained at the same time points. Inflammatory cytokine levels, presence, and quantities of 11 different bacterial species were determined. Smoking status was validated by cotinine assay.

Results: Fourteen smoker and 13 non-smoker patients completed the study protocol and revealed similar clinical findings except for the higher plaque scores in the non-smokers at 6months $(\mathrm{p}<0.01)$. Significant differences were found between the study groups in biofluid cytokine levels at 1-, 3-months $(\mathrm{p}<0.01)$. Gram-negative bacteria were more abundant in the smokers at baseline and so were Gram-positive bacteria in the non-smokers $(p<0.01)$. Gramnegative bacteria repopulated in the smokers faster than in the non-smokers $(\mathrm{p}<0.01)$.

Conclusion: The present findings suggest that smoker patients with periodontitis stage III and IV respond well to the non-surgical periodontal treatment during the 6-month follow-up. However, smokers exhibit faster repopulation of Gram-negative bacteria.

MeSH Key Words: Bacteria; cytokines; gingival crevicular fluid; periodontitis; smoking 


\section{INTRODUCTION}

Smoking is the major preventable risk factor in the initiation and progression of periodontal diseases. Smokers have more severe periodontal disease; more pocketing, more bone loss, more attachment loss, more gingival recession, and more susceptibility for tooth loss. ${ }^{1}$ Furthermore, smoking adversely affects the outcomes of non-surgical periodontal treatment. ${ }^{2}$ Less reduction in probing depth and clinical attachment gain have been found in smoker periodontitis patients compared to their non-smoker counterparts following non-surgical periodontal treatment. ${ }^{3}$ Smoker patients with aggressive periodontitis ( $\mathrm{AgP})$ have been reported not to respond well to mechanical non-surgical periodontal treatment. ${ }^{4}$ Accordingly, smoking has been associated with an increased risk for recurrence of periodontitis in AgP patients. ${ }^{5}$

$\mathrm{AgP}$ is characterized by attachment loss that is usually incompatible with the amount of plaque and dental calculus. ${ }^{6}$ Few studies have investigated the success of non-surgical periodontal therapy in AgP patients and it is often not possible to predict the prognosis of various treatment options. ${ }^{7}$ Rosalem et al. ${ }^{8}$ compared the response to non-surgical periodontal therapy in GAgP and generalized chronic periodontitis patients using clinical, immunologic and microbiological data and suggested that the treatment response was similar in both groups.

The interleukin-17 (IL-17) family is composed of six members (IL-17A-IL-17F). IL-17A, IL-17F homodimers and IL-17A/F heterodimer have been demonstrated to drive expression of antimicrobial peptides, cytokines, chemokines, and matrix metalloproteinases from fibroblasts, endothelial and epithelial cells and therefore, have pro-inflammatory properties. ${ }^{9}$ In contrast, IL-17E has been shown not only to be a driver of a T helper-2 immune response but also a suppresser of the destructive pro-inflammatory responses. ${ }^{10}$ Indeed, IL-17E has been shown to inhibit IL-17A and $P$. gingivalis induced nuclear factor-kappa B (NFKB) activity. ${ }^{10}$ Therefore, IL-17E is likely to have opposing biological effects to IL-17A, IL-17F and IL-17A/F. During the inflammatory response, pro-inflammatory cytokines such as IL-1 $\beta$, IL-6, IL-11, IL-17A, IL-17F and tumour necrosis factor-alpha (TNF- $\alpha$ ) can induce alveolar bone resorption by increasing the expression of receptor activator of NFKB ligand (RANKL) and reducing osteoprotegerin (OPG) production from osteoblasts and stromal cells. ${ }^{10}$ In a recent study, it was stated that systemic cytokine signaling via IL-17 is impaired in smokers and this impairment is associated with bacterial colonization by opportunistic pathogens locally in the airways. ${ }^{11}$ Many studies suggested that IL-17 has a significant role in the pathogenesis of periodontal diseases. ${ }^{12-}$ 14 
Smoking affects host cytokine levels in biofluids ${ }^{15}$ and pathopionts are more abundant in the subgingival microflora of smokers. ${ }^{16}$ However, the exact mechanisms of the detrimental effects of smoking on periodontal tissues remain unclear. ${ }^{17}$ It was hypothesised that non-smoker patients with GAgP will respond better in terms of the clinical, biochemical and microbiological parameters to the conventional mechanical non-surgical periodontal treatment compared to the smoker counterparts. Therefore, the aim of this study was to evaluate possible effects of smoking on the outcomes of non-surgical periodontal treatment.

\section{MATERIALS and METHODS}

\section{Study population}

A total of 48 Caucasian patients were screened and 34 patients with the clinical and the radiological diagnosis of GAgP were recruited for this study between June 2014 and August 2015. The diagnosis of GAgP was assigned in accordance with the clinical criteria stated in the consensus report of the World Workshop in Periodontitis. ${ }^{18}$ According to the consensus report published by Papapanou et al. ${ }^{19}$ the patients included in the present study comply with the definition of periodontitis Stage III or IV with regard to the extent and severity and Grade C due to the early onset of the disease. These patients had at least one site with probing depth (PD) and clinical attachment level (CAL) $\geq 5 \mathrm{~mm}$ in their incisors and/or first molars and at least six other teeth with similar PD and CAL measurements, and familial aggregation (all individuals were asked if they had any family member with current or history of severe periodontal disease). Smokers were those who reported smoking more than 10 cigarettes/day for more than 5 years, while non-smokers were those who reported that they had never smoked. Smoking status was validated with salivary cotinine analysis.

The study was approved by the Ethics Committee of Ege University, İzmir, Turkey (Protocol number; 14-9/28) and registered to the US National Institutes of Health Clinical Trials Registry site with the ID number of NCT03512938. Full accordance with ethical principles including the World Medical Association's Declaration of Helsinki as revised in 2000 was provided. The study protocol was explained and written informed consent was received from each individual before clinical periodontal examinations and sampling. Medical and dental histories were obtained. Exclusion criteria included presence of less than 15 teeth, known medical disorders such as diabetes mellitus, rheumatoid arthritis, immunological disorders and history of antibiotic, anti-inflammatory or periodontal treatment in the preceding 6 months. 


\section{Sample Size Calculation}

A two-sided two-sample t-test with a significance level of 5\% was used for the sample size calculation. Based on this analysis, a sample size of 12 subjects for each group was estimated to be necessary to achieve $80 \%$ power to detect a difference of $1 \mathrm{~mm}$ (SD 0.85) between the two groups in the mean PD reduction. A total of 34 were to be selected to compensate for a possible drop-out during the course of the study.

\section{Non-surgical periodontal therapy}

Patients were motivated and instructed to brush twice a day with the modified Bass technique and use interdental toothbrushes and dental floss once a day regularly. Each patient underwent quadrant-wise scaling and root planing (SRP) over a 4-week period. Root planing (RP) was performed under local anaesthesia (2\% lidocaine, epinephrine 1:100.000) and a standard curette set $^{*}$ newly sharpened with a stone ${ }^{\S}$ was used for each patient. A single researcher (BK) performed SRP in all patients. The procedures were continued until a hard, smooth root surface was sensed with an explorer tip.

\section{Clinical measurements}

Clinical periodontal measurements were performed by a calibrated periodontist (BK) at baseline and also 1-, 3-, and 6-months after completion of SRP. Saliva, serum, gingival crevicular fluid (GCF), and subgingival plaque samples were collected at the same time points. Calibration of the investigator was performed with measurements in five patients, who were diagnosed to have chronic periodontitis and were not included in this study. PD and CAL measurements were repeated with two days intervals. The intraexaminer kappa scores were 0.96 for PD and 0.85 for CAL. PD, CAL, bleeding on probing (BOP) (BOP deemed positive if bleeding occurred within 15 seconds after probing $)^{20}$, and plaque index (PI) ${ }^{21}$ were recorded at six sites of all teeth present using a manual probe. In each patient, one site with the greatest PD in each quadrant was selected for GCF and subgingival plaque sampling.

\section{Saliva and serum sampling}

\footnotetext{
+ Hu-Friedy, Chicago, IL.

$\S$ Arkansas Stone SS4, Hu-Friedy, Chicago, IL.
} 
In the morning, following an overnight fast, during which subjects were requested not to drink (except water) or to chew gum, whole saliva samples were obtained by expectorating into polypropylene tubes. The samples were immediately frozen and stored at $-40^{\circ} \mathrm{C}$ until the sample collection period was completed.

Nine milliliters of venous blood were taken from the antecubital vein by a standard venipuncture method and centrifuged for 10 minutes at $3000 \mathrm{rpm}$, separating serum from the cells. The serum samples were immediately frozen and stored at $-40^{\circ} \mathrm{C}$.

\section{GCF and subgingival plaque sampling}

GCF samples were obtained from buccal aspects of one interproximal site in each quadrant. Supragingival plaque was removed carefully by sterile curettes; the surfaces were dried and isolated by cotton rolls. Filter paper strips ${ }^{l}$ were placed in the orifice of the pocket for 30 seconds. Care was taken to avoid mechanical trauma, and strips contaminated with blood were discarded. The absorbed GCF volume was estimated by a calibrated instrument ${ }^{\text {Il }}$. Then, the strips were placed into polypropylene tubes and immediately frozen and stored at $-40^{\circ} \mathrm{C}$. GCF samples were converted to an actual volume $(\mu \mathrm{L})$ by reference to a standard curve.

Following GCF sample collection, subgingival plaque samples were collected from the same sites by sterile paper points ${ }^{\#}$. Paper points inserted into the deepest part of the pocket and kept for 5 seconds, were then placed into sterile propylene tubes, immediately frozen and stored at $-40^{\circ} \mathrm{C}$.

\section{Biochemical analyses}

Smoking status of each patient was determined by cotinine analysis in the salivary samples using an Enzyme Immunoassay (EIA) ${ }^{* *}$. GCF samples from each patient were eluted in $1 \mathrm{~mL}$ of phosphate-buffered saline containing $0.05 \%$ polysorbate surfactant ${ }^{\dagger \dagger}$ with a 60 -minute incubation on a rotary mixer at $+4^{\circ} \mathrm{C}$. Serum and saliva samples were used neat. Enzyme-linked immunosorbent assay (ELISA) kits were purchased for IL-17A, IL-17E, IL-17A/F, IL-6 $6^{\ddagger}$, IL-

\footnotetext{
II Periopaper, ProFlow, Amytyville, NY.

II Periotron 8000, Oraflow, Plainview, NY.

\# Diadent Group International, Seoul, Korea.

**Alere toxicology, London, UK.

$\dagger \dagger$ Tween 20, Sigma-Aldrich, Poole, UK.

t+ R\&D Systems, Abingdon, UK.
} 
$1 \beta$ and TNF- $\alpha^{\S \S}$ analysis. The ELISA assays were carried out on $50 \mu \mathrm{L}$ samples of GCF in duplicate and on $50 \mu \mathrm{L}$ of serum/saliva in triplicate according to the manufacturers' recommendations. The minimum detection limits in the assays were $1.9 \mathrm{pg} / \mathrm{mL}$ for IL-17A and IL-17E, $7.9 \mathrm{pg} / \mathrm{mL}$ for IL-17A/E, and $0.4 \mathrm{pg} / \mathrm{mL}$ for IL- $1 \beta$ and TNF- $\alpha$, and $0.8 \mathrm{pg} / \mathrm{ml}$ for IL-6. The results were expressed as picograms per 30 seconds for the total amounts in the samples and as picograms per milliliter for concentrations.

\section{Preparation and assessment of genomic DNA}

A Gram-positive DNA isolation kit"ll was used to prepare genomic DNA from the pelleted microbes present in the paperpoint sample and from known quantities of laboratory strains of target microorganisms. The DNA in standards and plaque samples was measured by fluorimetric analysis ${ }^{\text {IIII. }}$. DNA content of the target species was used to fix the copy number of its bacterial genome to use as a standard for each realtime quantitative polymerase chain reaction $(\mathrm{RTqPCR})$ assay.

\section{RT-qPCR}

Real-time reagents were purchased from ${ }^{\# \#, * * *}$ and previously validated assays ${ }^{22}$ were used for detection and quantification of bacterial cell copy numbers in $1 \mu \mathrm{g}$ plaque DNA for the following bacteria: P.gingivalis, A. actinomycetemcomitans, P. intermedia, P. micros, T. forsythia, F. nucleatum T. denticola S. mutans, F. nucleatum, S. oralis, A. naeslundi, V. dispar. The PCR reaction was performed on a thermocycler ${ }^{\dagger \dagger}$, under the following conditions: 10 minutes at $95^{\circ} \mathrm{C}$ and 40 cycles of 30 seconds at $95^{\circ} \mathrm{C}$ and 1 minute at $60^{\circ} \mathrm{C}$. PCR assay efficiency was determined as follows: efficiency $(E)=10(-1 /$ slope $)-1$ and deemed to be acceptable (between 91\% and 104\%). None of the primer amplified DNA purified from microbial standards other than the target species.

\section{Statistical analysis}

$\S \S$ Thermo Fisher Renfrew, UK.

|||| Masterpure, Illumina, San Diego, CA.

III CyQUANT assay system, Invitrogen, Thermo Fisher Scientific, Waltham, MA.

\#\# Thermo Fisher Scientific, Paisley, UK.

*** Eurogentech, Liege, Belgium.

$\dagger \dagger \dagger$ MRX3000 Agilent Thermocycler, Agilent, Edinburgh, UK. 
Microsoft Excel and commercially available statistical softwares ${ }^{\dagger+}, \S \S$ were used to analyse the data. Sample size calculation included an estimated alpha error of $1 \%$ to allow for multiple testing of two treatment groups (smokers and non-smokers) and beta error of $20 \%$ with an effect size $=1$ for differences in the levels of biomarkers or copy numbers of several putative pathogens. For a two-sided test, a minimum of 27 subjects were required (13 in one group and 14 in the other group) for a longitudinal study a minimum of 34 (17 per group) were selected to compensate for possible drop-outs during the course of the study and allow for initial crosssectional comparisons in an explorative study design. It was anticipated that larger effect sizes of 1.5 would be required in analyses where multiple testing was considered in cross-sectional analysis if there were any drop-outs from the study participants and alpha $=0.01$.

Only the data of those patients who completed the entire study protocol were included in the longitudinal analyses. Cross-sectional analyses were performed on all available participants at each time point (see Fig. 1). Mean values of PD, CAL, and PI measurements were calculated for each patient and BOP was presented as percentages. CAL, PD, BOP, and PI data were compared between the study groups using Mann Whitney-U test. The Friedman test and Dunn's multiple comparison test was used for post hoc comparisons of the repeat sample tests. Although it was not relevant to the outcome, the baseline relationship between clinical, biochemical, and microbiological data was evaluated by Spearman correlation analysis.

\section{RESULTS}

\section{Clinical measurements}

Four smoker and three non-smoker patients had to be excluded from the study due to their noncompliance with appointments. The study was completed with 13 non-smoker patients (9 females, 4 males) aged between 23-38 years (mean age 31.00 \pm 4.90$)$ and 14 smoker patients (4 females, 10 males) aged between 26-38 years (mean age 32.93 \pm 3.37 ). The study groups differed in gender distribution $(\mathrm{p}<0.05)$, but were similar in mean ages. The patients' medical and dental histories, as well as socio-economical states were similar in the smoker and non-smoker groups. All patients had middle-income levels on the study registration forms and reported 12-14 years of education. The mean value of salivary cotinine concentration was $37.65 \mathrm{ng} / \mathrm{ml}$ in the smoker

HH SPSS Inc. version 21 IBM, Chicago, IL. $\S \S \S$ Graphpad Prism version 5, La Jolla. 
group (min-max; 32.92-50.12 $\mathrm{ng} / \mathrm{ml}$ ) and $0.65 \mathrm{ng} / \mathrm{ml}$ in the non-smoker group (min-max; 0.48$0.76 \mathrm{ng} / \mathrm{ml})(\mathrm{p}<0.001)$ (Table 1). Smoking habits of the patients did not change during the study.

At baseline, the clinical periodontal measurements were similar in the smoker and nonsmoker patient groups (Table 2). At 1-month, PD, CAL, and BOP decreased significantly in the smoker group compared to the baseline $(\mathrm{p}<0.0001)$. The 3-and 6-month evaluations revealed significant reductions in all clinical periodontal parameters compared to the baseline values in both the smoker and non-smoker patient groups ( $\mathrm{p}<0.0001)$. At 1-month, both groups revealed significant decreases in the number of sites with PD> $6 \mathrm{~mm}$ compared to the baseline $(\mathrm{p}<0.0001)$. Only the non-smoker group exhibited significant decreases in the counts and percentages of sites with PD $>6 \mathrm{~mm}$ at 3 -month compared to the 1-month $(\mathrm{p}<0.0001)$ (Table 2).

\section{Biochemical analysis}

Intergroup comparisons (i.e., between baseline and follow-up) revealed no statistically significant differences in serum IL-17A, IL-17E, IL-1 $\beta$, TNF- $\alpha$, IL-6 cytokine levels between the groups at majority of the time points (Fig. 1). At 1-month, IL-17E levels were higher in the non-smoker group $(\mathrm{p}<0.01)$. At 6-month, IL-17A levels were higher in the smoker group $(\mathrm{p}<0.01)$. Intragroup comparisons (i.e., between smokers and non-smokers) showed that TNF$\alpha$ and IL-6 decreased significantly at 1 -month in both groups $(\mathrm{p}<0.0001)$. At 3 -month IL-1 $\beta$ decreased only in the smoker group $(\mathrm{p}<0.0001)$, while both TNF- $\alpha$ and IL-6 exhibited significant decreases compared to the baseline values ( $\mathrm{p}<0.0001)$. At 6-month, only IL-6 levels decreased significantly in both groups $(\mathrm{p}<0.0001)$.

There were no intergroup differences in salivary cytokine levels at baseline (Fig. 2). At 1 -month, IL-17A and IL-1 $\beta$ levels were higher in the smoker group $(\mathrm{p}<0.01)$. At 3 -month IL$1 \beta$, and IL-6 levels were higher in the non-smoker group $(\mathrm{p}<0.01)$.

The GCF sample volumes were similar in the smoker and non-smoker groups at baseline and decreased significantly in both groups at 1-, 3-, and 6-months $(\mathrm{p}<0.0001)$ (Table 1). At baseline, GCF cytokine levels were similar (Fig. 3). At 1-month; IL-17A levels were higher in the non-smoker group $(\mathrm{p}<0.01)$. There was no significant difference between the two groups in the 3-month GCF data ( $>>0.05)$.

\section{Microbiological analysis}

At baseline, copy numbers of P.gingivalis, P.intermedia, T.denticola and T. forsythia (Fig. 4) were higher in the smoker group $(\mathrm{p}<0.01)$. Gram-positive bacteria such as $S$. oralis were higher 
in the non-smoker group ( $\mathrm{p}<0.01)$ (Fig. 5). The frequency of carriage did not differ between smokers and non-smokers (data not shown). At 1-month, and at 3-month, the copy numbers of all bacteria were similar in both groups. At 6-month copy numbers of $F$. nucleatum, $P$. gingivalis, $P$. intermedia, S. oralis, $T$. denticola and $T$. forsythia were significantly higher in the smoker group $(\mathrm{p}<0.01)$. Similar results were obtained when the proportions of each organisms to the total bacterial DNA content were compared (data not shown). Total bacterial counts at 3and 6-months were similar in both groups and decreased significantly from baseline to 1-, 3-, and 6-months ( $\mathrm{p}<0.0001)$ (data not shown).

\section{Spearman correlations}

At baseline: although there were associations between clinical data (such as, PD, CAL, the number of sites with PD >5mm) and levels of certain cytokines TNF- $\alpha$, IL-17A, IL-6 in serum and saliva (all Rho >0.32, p<0.05), levels of TNF- $\alpha$, IL-17A, and IL-6 in GCF (all Rho>0.32, $\mathrm{p}<0.05)$ and several correlations were observed between IL-1 $\beta$, TNF- $\alpha$, IL-6 and IL-17A and microbiological parameters (all Rho>0.333, $\mathrm{p}<0.05$ ). As anticipated these relationships were not observed following treatment.

\section{DISCUSSION}

Smoking is the most common modifiable risk factor for periodontitis worldwide and it is among the major determining factors for the grade of periodontitis in the 2017 classification. ${ }^{19}$ In the present study, the response to mechanical non-surgical periodontal treatment was evaluated comparatively in smoker and non-smoker patients with periodontitis stage III or IV, and grade $\mathrm{C}$ using clinical, biochemical and microbiological parameters. Baseline clinical periodontal status is of upmost importance when comparing the response to treatment among two or more study groups. The present study groups revealed similar baseline clinical findings in terms of oral hygiene, gingival inflammation and visual signs of tissue destruction. Moreover, the groups were comparable with regard to the socio-economic status and educational attainment. After non-surgical periodontal treatment, the clinical periodontal measurements decreased similarly in both smoker and non-smoker patients.

Potential biomarkers for monitoring periodontal diseases may be sought in different biofluids; saliva, GCF, or serum. All these media have their own benefits; GCF provides sitespecific data, whereas saliva reflects the oral health as a whole and serum gives information 
also about the systemic health or environmental factors such as smoking. In the present study, all three biofluid samples were investigated to have a comprehensive information.

Adverse effects of smoking on clinical healing have been reported in previous studies ${ }^{23-28}$. Hughes et al. ${ }^{4}$ reported that smoker patients with GAgP did not respond well to non-surgical periodontal treatment. Smokers have been reported to have a higher risk for unresponsive pockets and further breakdown during supportive periodontal treatment. ${ }^{2}$ Nonsmoker patients with periodontitis exhibited better healing responses than smokers. ${ }^{16}$ However, another study reported contradicting data as both non-smokers and smokers exhibited similarly significant reductions in PD and bleeding scores between baseline and 6 months after nonsurgical periodontal treatment. ${ }^{29}$ Accordingly, the present study indicated similar outcomes for $\mathrm{CAL}$ and BOP in the smoker and non-smoker groups.

Some studies suggest that the use of antibiotics in the treatment of aggressive periodontitis provides short-term benefits. ${ }^{30-32}$ Considering the potential side-effects ${ }^{33}$ together with limited benefits of short-term duration, no additional antibiotics were used in the present study.

Suzuki et al. ${ }^{34}$ found no correlation between GCF IL-1 $\beta$ levels and clinical parameters in $\mathrm{AgP}$ and the current study partly agreed with their results. Serum IL-17 affects the production of IL-1, IL-6 and TNF- $\alpha$, and this cytokine was increased in AgP patients compared to healthy individuals and it was suggested to play a role in the pathogenesis of AgP. ${ }^{12}$ GCF IL-17A levels were found to be significantly elevated in patients with AgP. ${ }^{13} \mathrm{GAgP}$ patients exhibited higher GCF IL-11:IL-17A ratio than healthy controls and this has been suggested to have a role in the pathogenesis of the disease. ${ }^{14}$ Teles et al., ${ }^{35}$ also reported increased GCF levels of IL-1 $\beta: I L-10$ in patients with GAgP. On the other hand, patients with AgP have been shown to have higher serum and salivary IL- $1 \beta$ and pentraxin-3 concentrations than the healthy individuals. ${ }^{36}$ Similar findings have been reported for salivary TNF- $\alpha$ levels. ${ }^{28}$ In another study, serum concentrations of TNF- $\alpha$, IL-4, IL-17A and IL-23 have been investigated in AgP patients at baseline and at 6months after SRP. ${ }^{37}$ The authors have documented higher IL-17A and TNF- $\alpha$ levels in AgP patients than those of healthy subjects. Moreover, TNF- $\alpha$ levels remained high in GAgP patients after treatment.

De Lima Oliveira et al. ${ }^{38}$ stated that the IL-1 $\beta$ :IL-10 ratio may play an important role in the pathogenesis of AgP. Moreover, IL-17A, IL-23 and myeloperoxidase maintained high GCF levels in GAgP after SRP. ${ }^{39}$ Serum IL-17E levels have been suggested as a good indicator of 
favourable treatment outcome in periodontitis patients after scaling and root-planing. ${ }^{40}$ Rosalem et $\mathrm{al}^{8}{ }^{8}$ found that GCF IL-1 $\beta$ levels decreased significantly 3 months after therapy in GAgP patients. Another study reported that GCF concentrations of IL-6 and TNF- $\alpha$ decreased from baseline to the 6-month in non-smokers as well as smokers. ${ }^{29}$ Although similar trends were observed, the current study agreed only in part with the earlier investigations presumably because of individual differences or by the complex nature of GAgP and possible presence of disease subgroups. This might be explained by the transient post-treatment falls and increases observed; paticularly for TNF- $\alpha$. In the current study, IL-17E levels in biofluids other than serum decreased following non-surgical periodontal treatment. The reason for this difference remains obscure. However, other key cytokines such as TNF- $\alpha$ and IL-17A are increased in these biofluids and this may have a direct impact on local production of IL-17E. One might also speculate that the increase in pro-inflammatory cytokines TNF- $\alpha$ and IL-17A in GCF and saliva appears to coincide with a re-colonization of the tissue by pathogenic microorganisms. Correlations between several Gram-negative organisms and pro-inflammatory molecules were evident particularly at baseline, these associations were weaker during the follow-up after SRP, but this was probably due to the reduction in the number study participants as well as to the reduction in biomarker and bacterial counts.

Significant positive correlations between PD, CAL, $P$. gingivalis and $T$. forsythia have been reported in GAgP patients. ${ }^{41}$ However, the current study indicated few correlations between the microorganisms and the clinical indices, and that these were only obseved at baseline. The major finding of the present study is that potentially pathogenic Gram-negative bacteria copy numbers were significantly higher in the smokers. In contrast to the smokers, non-smoker patients revealed higher copy numbers of Gram-positive bacteria. The present findings are in line with those of Darby et al. ${ }^{16}$ in terms of significantly higher detection rates of $A$. actinomycetemcomitans and $P$. intermedia in smoker patients than the non-smoker ones. While the results of the current study are not strictly analogous; the smoker group exhibited significantly higher copy numbers of $P$. intermedia, $P$. gingivalis, $T$. denticola and $T$. forsythia at baseline while the carriage frequencies were similar. The present finding of lower baseline proportions of $P$. gingivalis in non-smokers is parallel to the previous studies showing that smokers are more likely to harbor higher numbers of $P$. gingivalis, and to exhibit more persistent infection. ${ }^{42,43}$ Copy numbers of A. actinomycetemcomitans were higher in the smoker group at 1- and 6-months after treatment and the copy numbers of $P$. gingivalis, P.intermedia, T. denticola and T. forsythia were higher in the smoker group at 6-months. In the smoker 
patients, carriage rates decreased following non-surgical periodontal treatment but incresed to the baseline levels by the 6-month reassessment and the copy numbers of Gram-negative bacteria increased to a greater extent in the smokers.

The major limitation of the present study is the rather low number of patients mainly due to the stringent inclusion and exclusion criteria and the dropout of patients during the followup period which resulted in some of the later cross-group comparisons having less than $80 \%$ statistical power. While the longitudinal component of the study retained sufficient statistical power (at an effect size $=1$ ) the follow-up period was shorter than desired because of the failure of sufficient patients to return at later time points. Another limitation may be the lack of blinding to the study group during the measurement of the clinical periodontal parameters as one researcher performed the SRP and clinical recordings in all patients. However, stainings on the teeth, increased gingival pigmentation, and the characteristic cigarette smell make blinding of the clinician extremely difficult if not impossible in studies that compare smokers and nonsmoker counterparts.

\section{CONCLUSION}

Within the limits of the present study, it may be concluded that smoker patients with periodontitis stage III or IV and grade C exhibit higher detection rates and faster recolonisation of Gram-negative bacteria in subgingival plaque samples. Further studies on a larger scale and longer follow-up periods are warranted to support the present findings.

\section{CONFLICTS OF INTEREST}

The authors have stated explicitly that there are no conflicts of interest in connection with this article.

ACKNOWLEDGEMENTS This study was supported by a grant from the Ege University Research Foundation (Project No: 2014 DIS 013) and funds from the University of Glasgow. 


\section{REFERENCES}

1. Dietrich T, Walter C, Oluwagbemigun K, Bergmann M, Pischon N. Boeing H. Smoking, smoking cessation, and risk of tooth loss: The EPIC-Potsdam study. J Dent Res. 2015; 94: 1369-1375.

2. Bunaes DF, Lie SA, Astrom AN, Mustafa K, Leknes KN. Site-specific treatment outcome in smokers following 12 months of supportive periodontal therapy. J Clin Periodontol. 2016; 43: 1086-1093.

3. Haesman L, Stacey F, Preshaw PM, McCracken GI, Hepburn S, Haesman PA. The effect of smoking on periodontal treatment response: a review of clinical evidence. J Clin Periodontol. 2006; 33: 241-253.

4. Hughes FJ, Syed M, Koshy B, et al. Prognostic factors in the treatment of generalized aggressive periodontitis: I. Clinical features and initial outcome. J Clin Periodontol. 2006; 33: 663-670.

5. Baumer A, El Sayed N, Kim TS, Reitmeir P, Eickholz P, Pretzl B. Patient-related risk factors for tooth loss in aggressive periodontitis after active periodontal therapy. J Clin Periodontol. 2011; 38: 347-354.

6. Meyer J, Lallam-Laroye C, Dridi M. Aggressive periodontitis - What exactly is it? J Clin Periodontol. 2004; 31: 586-587.

7. Teughels W, Dhondt R, Dekeyser C, Quirynen M. Treatment of aggressive periodontitis. Periodontol 2000. 2014;65:107-133.

8. Rosalem W, Rescala B, Teles RP, Fischer RG, Gustafsson A, Figueredo CM. Effect of non-surgical treatment on chronic and aggressive periodontitis: Clinical, immunologic, and microbiologic findings. J Periodontol. 2011; 82: 979-989.

9. Jin W, Dong C. IL-17 cytokines in immunity and inflammation. Emerg Microbes Infect. 2013; 2: 60. Doi: 10.1038/emi.2013.58

10. Awang RA, Lappin DF, MacPherson A, et al. Clinical associations between IL-17 family cytokines and periodontitis and potential differential roles for IL-17A and IL-17E in periodontal immunity. Inflamm Res. 2014; 63: 1001-1012

11. Andelid K, Tengvall S, Andersson A, et al. Systemic cytokine signaling via IL-17 in smokers with obstructive pulmonary disease: a link to bacterial colonization? Int J Chron Obstruct Pulmon Dis. 2015;10: 689-702.

12. Schenkein HA, Koertge TE, Brooks CN, Sabatini R, Purkall DE, Tew JG. IL-17 in sera from patients with aggressive periodontitis. J Dent Res. 2010; 89: 943-947. 
13. Shaker OG, Ghallab NA. IL-17 and IL-11 GCF Levels in aggressive and chronic periodontitis patients: Relation to PCR bacterial detection. Mediators Inflamm. 2012; 2012: 174764. Doi: $10.1155 / 2012 / 174764$

14. Ay ZY, Y1lmaz G, Özdem M, et al. The gingival crevicular fluid levels of interleukin-11 and interleukin-17 in patients with aggressive periodontitis. J Periodontol. 2012; 83: 1425 1431.

15. Boström L, Linder LE, Bergström J. Smoking and crevicular fluid levels of IL-6 and TNFalpha in periodontal disease. J Clin Periodontol. 1999; 26: 352-357.

16. Darby IB, Hodge PJ, Riggio MP, Kinane DF. Clinical and microbiological effect of scaling and root planing in smoker and non-smoker chronic and aggressive periodontitis patients. J Clin Periodontol. 2005;32:200-206.

17. Buduneli N, Kinane DF. Host-derived diagnostic markers related to soft tissue destruction and bone degradation in periodontitis. J Clin Periodontol. 2011; 38: 85-105.

18. Armitage GC. Development of a classification system for periodontal diseases and conditions. Ann Periodontol. 1999; 4: 1-6.

19. Papapanou PN, Sanz M, Buduneli N, et al. Periodontitis: Consensus report of workgroup 2 of the 2017 World Workshop on the Classification of Periodontal and Peri-Implant Diseases and Conditions. J Periodontol. 2018; 89: Suppl 1: S173-S182

20. O'Leary TJ, Drake RB, Naylor JE. The plaque control record. J Periodontol. 1972; 43: 38.

21. Quigley GA, Hein JW. Comparative cleansing efficiency of manual and power brushing. J Am Dent Assoc. 1962; 65: 26-29.

22. Akcalı A, Güven Yılmaz S, Lappin DF, Eğrilmez S, Buduneli, N. Effect of gingival inflammation on the inflammatory response in patients with idiopathic uveitis. J Clin Periodontol. 2016; 43: 637-645.

23. Armitage GC, Cullinan MP, Seymour GJ. Comparative biology of chronic and aggressive periodontitis: Introduction. Periodontol 2000. 2010; 53: 7-11.

24. Duarte PM, Bastos MF, Fermiano D, et al. Do subjects with aggressive and chronic periodontitis exhibit a different cytokine/chemokine profile in the gingival crevicular fluid? A systematic review. J Periodontal Res. 2015; 50: 18-27.

25. Picolos DK, Lerche-Sehm J, Abron A, Fine JB, Papapanou PN. Infection patterns in chronic and aggressive periodontitis. J Clin Periodontol. 2005;32:1055-1061.

26. Ryder MI. Comparison of neutrophil functions in aggressive and chronic periodontitis. Periodontol 2000. 2010; 53: 124-137.

27. Stabholz A, Soskolne WA, Shapira L. Genetic and environmental risk factors for chronic 
periodontitis and aggressive periodontitis. Periodontol 2000. 2010; 53: 138-153.

28. Varghese S, Thomas H, Jayakumar N, Sankari M, Lakshmanan R. Estimation of salivary tumor necrosis factor-alpha in chronic and aggressive periodontitis patients. Contemp Clin Dent. 2015; 6: 152. Doi: 10.4103/0976-237X.166816

29. De Genaro Modanese D, Tiosso-Tamburi R, Furletti de Goes VF, et al. Clinical and immunoinflammatory evaluation of one-stage full-mouth ultrasonic debridement as a therapeutic approach for smokers with generalized aggressive periodontitis: A short-term follow-up study. J Periodontol. 2016; 87: 1012-1021.

30. Mestnik MJ, Feres M, Figueiredo LC, Duarte PM, Lira EAG, Faveri M. Short-term benefits of the adjunctive use of metronidazole plus amoxicillin in the microbial profile and in the clinical parameters of subjects with generalized aggressive periodontitis. J Clin Periodontol. 2010; 37: 353-365.

31. Keestra JAJ, Grosjean I, Coucke W, Quirynen M, Teughels W. Non-surgical periodontal therapy with systemic antibiotics in patients with untreated aggressive periodontitis: a systematic review and meta-analysis. J Periodontal Res. 2015; 50: 689-706.

32. Varela VM, Heller D, Silva-Senem MX, Torres MC, Colombo AP, Feres-Filho EJ. Systemic antimicrobials adjunctive to a repeated mechanical and antiseptic therapy for aggressive periodontitis: a 6-month randomized controlled trial. J Periodontol. 2011; 82: 1121-1130.

33. Eickholz P, Dannewitz B, Kim TS. Antibiotics in periodontal therapy. Perio 2005; 2: 235251.

34. Suzuki M, Ishihara Y, Kamiya Y, et al. Soluble interleukin-1 receptor type II levels in gingival crevicular fluid in aggressive and chronic periodontitis. $J$ Periodontol. 2008; 79: 495-500.

35. Teles RP, Gursky LC, Faveri M, et al. Relationships between subgingival microbiota and GCF biomarkers in generalized aggressive periodontitis. J Clin Periodontol. 2010; 37: 313323.

36. Gümüş P, Nizam N, Nalbantsoy A, Özçaka Ö, Buduneli N. Saliva and serum levels of pentraxin-3 and interleukin-1 $\beta$ in generalized aggressive or chronic periodontitis. $J$ Periodontol. 2014; 85: 40-46.

37. Duarte PM, da Rocha M, Sampaio E, et al. Serum levels of cytokines in subjects with generalized chronic and aggressive Periodontitis before and after non-surgical periodontal therapy: A pilot study. J Periodontol. 2010; 81: 1056-1063.

38. De Lima Oliveira AP, De Faveri M, Gursky LC, et al. Effects of periodontal therapy on 
GCF cytokines in generalized aggressive periodontitis subjects. J Clin Periodontol. 2012; 39: 295-302.

39. Çifçibaşı E, Koyuncuoğlu C, Çıblak M, et al. Evaluation of local and systemic levels of interleukin-17, interleukin-23, and myeloperoxidase in response to periodontal therapy in patients with generalized aggressive periodontitis. Inflamm. 2015; 38: 1959-1968.

40. Nile CJ, Apatzidou DA, Awang RA, Riggio MP, Kinane DF, Lappin DF. The effect of periodontal scaling and root polishing on serum IL-17E concentrations and the IL-17A:IL17E ratio. Clin Oral Investig. 2016; 20: 2529-2537.

41. Tomita S, Komiya-Ito A, Imamura K, et al. Prevalence of Aggregatibacter actinomycetemcomitans, Porphyromonas gingivalis and Tannerella forsythia in Japanese patients with generalized chronic and aggressive periodontitis. Microb Pathog. 2013; 6162: 11-15.

42. Grossi SG, Goodson JM, Gunsolley JC, et al. Mechanical therapy with adjunctive minocycline microspheres reduces red-complex bacteria in smokers. J Periodontol. 2007; 78: $1741-1750$.

43. Bagaitkar J, Lisa R, Williams LR, et al. Tobacco-induced alterations to Porphyromonas gingivalis-host interactions. Environ Microbiol. 2009; 11: 1242-1253. 


\section{Figure legends}

Figure 1. Cytokine levels in serum of smokers and non-smokers with aggressive periodontitis before and after non-surgical therapy. A. Serum IL-17A Level Changes B. Serum IL-17E Level Changes C. Serum IL-17A/E Level Changes D. Serum IL-1 $\beta$ Level Changes E. Serum TNF- $\alpha$ Level Changes F. Serum IL-6 Level Changes

${ }^{*}$ Significant difference between smokers and non-smokers $(\mathrm{p}<0.01){ }^{\dagger}$ Significant difference from baseline data in the group $(\mathrm{p}<0.0001)$. ${ }^{+}$Significant difference from 1 -month data in the group $(p<0.0001) .{ }^{8}$ Significant difference from 3 -month data in the group $(p<0.0001)$.

Figure 2. Cytokine levels in saliva of smokers and non-smokers with aggressive periodontitis before and after non-surgical therapy. A. Saliva IL-17A Level Changes B. Saliva IL-17E Level Changes C. Saliva IL-17A/E Level Changes D. Saliva IL-1 $\beta$ Level Changes E. Saliva TNF- $\alpha$ Level Changes F. Saliva IL-6 Level Changes

*Significant difference between smokers and non-smokers $(\mathrm{p}<0.01){ }^{\dagger}$ Significant difference from baseline data in the group $(\mathrm{p}<0.0001)$. Significant difference from 1-month data in the group $(\mathrm{p}<0.0001) .{ }^{\S}$ Significant difference from 3 -month data in the group $(\mathrm{p}<0.0001)$.

Figure 3. Cytokine levels in GCF of smokers and non-smokers with aggressive periodontitis before and after non-surgical therapy. A. GCF IL-17A Level Changes B. GCF IL-17E Level Changes C. GCF IL17A/E Level Changes D. GCF IL-1 $\beta$ Level Changes E. GCF TNF- $\alpha$ Level Changes F. GCF IL-6 Level Changes

*Significant difference between smokers and non-smokers $(p<0.01){ }^{\dagger}$ Significant difference from

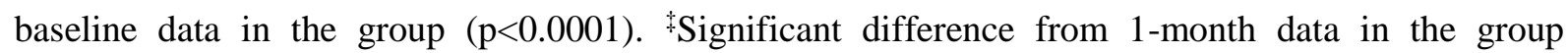
$(\mathrm{p}<0.0001) .{ }^{\S}$ Significant difference from 3 -month data in the group $(\mathrm{p}<0.0001)$.

Figure 4. Real-time PCR detection of Copy numbers of DNA from Gram negative bacteria. in the dental plaque of smokers and nonsmokers with Aggressive periodontitis before and after non-surgical therapy. A) A. actinomycetemcomitans, B) F. nucleatum,C) $P$. gingivalis, D) $P$. intermedia, E) $P$. oralis, F) $T$. denticola, G) T. forsythia, $\mathrm{H}) \mathrm{V}$. dispar.

${ }^{*}$ Significant difference between smokers and non-smokers $(\mathrm{p}<0.01){ }^{\dagger}$ Significant difference from baseline data in the group $(\mathrm{p}<0.0001)$. ${ }^{ \pm}$Significant difference from 1-month data in the group $(\mathrm{p}<0.0001) .{ }^{\S}$ Significant difference from 3 -month data in the group $(\mathrm{p}<0.0001)$.

Figure 5. Real-time PCR detection of Copy numbers of DNA from Gram positive bacteria in the dental plaque of smokers and nonsmokers with Aggressive periodontitis before and after non-surgical therapy. A) A. naeslundii, B) S. mitis, C) S. oralis.

"Significant difference between smokers and non-smokers $(\mathrm{p}<0.01){ }^{\dagger}$ Significant difference from baseline data in the group $(\mathrm{p}<0.0001)$. Significant difference from 1-month data in the group $(\mathrm{p}<0.0001) .{ }^{\S}$ Significant difference from 3-month data in the group $(\mathrm{p}<0.0001)$. 
Table 1. Demographic findings in the study groups.

\begin{tabular}{lcc}
\hline Demographic Variable & $\begin{array}{c}\text { Non-smoker } \\
\text { Group }\end{array}$ & Smoker Group \\
\hline Patient count (n) & 13 & 14 \\
\hline Gender (female/ male) & $9 / 4$ & $4 / 10$ \\
\hline $\begin{array}{l}\text { Age (year) (min - max) } \\
\text { Age (year) } \\
\text { (Mean } \pm \text { SD) }\end{array}$ & $23-38$ & $26-38$ \\
\hline $\begin{array}{l}\text { Cigarette consumption/day } \\
(\text { median (Q1-Q3)) }\end{array}$ & $31.00 \pm 4.90$ & $32.93 \pm 3.37$ \\
\hline $\begin{array}{l}\text { Pack-Year } \\
(\text { Mean } \pm \text { SD) }\end{array}$ & $0(0-0)$ & $15(10-20)$ \\
\hline $\begin{array}{l}\text { Cotinine } \\
(\text { ng/ml }(\text { median }(\mathrm{Q} 1-Q 3))\end{array}$ & $0.65(0.48-0.76)$ & $37.65(32.92-50.12)$ \\
\hline
\end{tabular}


Table 2. Clinical periodontal findings in the study groups. All data are expressed as median (Q1-Q3) unless otherwise noted.

\begin{tabular}{|c|c|c|c|}
\hline Clinical variable & Time & Non-smokers $(n=13)$ & Smokers (n=14) \\
\hline \multirow{4}{*}{$\begin{array}{l}\text { PD (full mouth) } \\
(\mathrm{mm})\end{array}$} & Baseline & $4.19(3.89-4.89)$ & $4.43(4.35-5.20)$ \\
\hline & 1. month & $2.72(2.52-3.50)$ & $3.06(2.87-3.48)^{*}$ \\
\hline & 3. month & $2.59(2.40-3.26)^{*}$ & $2.89(2.74-3.41)^{*}$ \\
\hline & 6. month & $2.58(2.26-3.16)^{*}$ & $2.98(2.82-3.27)^{*}$ \\
\hline \multirow{4}{*}{$\begin{array}{l}\text { CAL (full mouth) } \\
(\mathrm{mm})\end{array}$} & Baseline & $4.85(4.28-5.02)$ & $5.42(4.40-6.25)$ \\
\hline & 1. month & $4.18(3.24-4.38)$ & $4.57(3.24-5.29)^{*}$ \\
\hline & 3. month & $3.82(3.09-4.21)^{*}$ & $4.10(3.11-4.87)^{*}$ \\
\hline & 6. month & $3.70(2.91-4.14)^{* \dagger}$ & $3.81(3.29-5.16)^{*}$ \\
\hline \multirow{4}{*}{$\begin{array}{l}\text { BOP (full mouth) } \\
(\%)\end{array}$} & Baseline & $79.76(56.94-100.00)$ & $66.87(52.28-94.07)$ \\
\hline & 1. month & $19,15(15,48-24,01)$ & $18,83(9,17-26,60)^{*}$ \\
\hline & 3. month & $14.88(12.90-17.76)^{*}$ & $15.91(9.68-18.00)^{*}$ \\
\hline & 6. month & $15.48(8.83-17.26)^{*}$ & $18.10(7.86-21.83)^{*}$ \\
\hline \multirow{4}{*}{$\begin{array}{l}\text { PI (full mouth) } \\
(0-5)\end{array}$} & Baseline & $3.28(3.12-3.59)$ & $3.47(3.28-3.57)$ \\
\hline & 1. month & $2.43(2.29-2.65)^{*}$ & $2.47(2.23-2.63)^{*}$ \\
\hline & 3. month & $2.45(2.21-2.60)^{*}$ & $2.31(2.13-2.50)^{*}$ \\
\hline & 6. month & $2.48(2.37-2.58)^{*}$ & $2.19(2.11-2.44)^{*}$ \\
\hline \multirow{4}{*}{$\begin{array}{l}\text { PD (sample sites) } \\
(\mathrm{mm})\end{array}$} & Baseline & $8.5(7.25-9.50)$ & $8.38(7.44-9.69)$ \\
\hline & 1. month & $4(3.30-6.50)^{*}$ & $4.5(4.25-5.63)^{*}$ \\
\hline & 3. month & $4(3.75-5.50)^{*}$ & $4.63(4.00-5.13)^{*}$ \\
\hline & 6. month & $3.75(3.25-4.50)^{*}$ & $3.88(3.50-4.25)^{*}$ \\
\hline \multirow{4}{*}{$\begin{array}{l}\text { CAL (sample sites) } \\
(\mathrm{mm})\end{array}$} & Baseline & $9(7.25-10.00)$ & $9(7.00-10.00)$ \\
\hline & 1. month & $5(4.00-8.00)^{*}$ & $6(4.00-8.00)^{*}$ \\
\hline & 3. month & $5(3.25-7.00)^{*}$ & $5.50(4.00-7.00)^{*}$ \\
\hline & 6. month & $4(3.00-6.75)^{*}$ & $5(4.00-6.25)^{*}$ \\
\hline \multirow{4}{*}{$\begin{array}{l}\text { PD (sites with PD> } 6 \text { mm) } \\
\text { (n) }\end{array}$} & Baseline & $21.00(10.50-47.50)$ & $29.00(17.00-41.50)$ \\
\hline & 1. month & $0.00(0.00-24.00)^{*}$ & $2.50(0.00-17.00)^{*}$ \\
\hline & 3. month & $0.00(0.00-12.50)^{* \dagger \dagger}$ & $2.00(0.00-8.00)^{*}$ \\
\hline & 6. month & $0.00(0.00-8.00)^{* \dagger}$ & $0.50(0.00-3.00)^{* \dagger \S}$ \\
\hline \multirow{4}{*}{$\begin{array}{l}\text { PD (sites with PD> } 6 \mathrm{~mm}) \\
(\%)\end{array}$} & Baseline & $13.46(6.48-26.47)$ & $17.90(11.22-34.29)$ \\
\hline & 1. month & $0.00(0.00-14.29)^{*}$ & $1.93(0.00-10.32)^{*}$ \\
\hline & 3. month & $0.00(0.00-8.01)^{* \dagger}$ & $1.26(0.00-5.55)^{*}$ \\
\hline & 6. month & $0.00(0.00-4.97)^{*+\S}$ & $0.33(0.00-1.84)^{* \dagger \S}$ \\
\hline \multirow{4}{*}{ GCF volume $(\mu \mathrm{l})$} & Baseline & $1(0.67-1)^{*}$ & $0.71(0.48-1)$ \\
\hline & 1. month & $0.5(0.24-0.67)^{*}$ & $0.42(0.18-0.75)^{*}$ \\
\hline & 3. month & $0.46(0.29-0.66)^{*}$ & $0.52(0.18-0.8)^{*}$ \\
\hline & 3. month & $0.51(0.28-0.66)^{*}$ & $0.45(0.12-0.74)^{*}$ \\
\hline
\end{tabular}

\footnotetext{
"Significantly lower than the baseline value $(\mathrm{p}<0.0001)$.

${ }^{+}$Significantly lower than the 1-month value $(\mathrm{p}<0.0001)$.

* Significantly difference between the study groups $(\mathrm{p}<0.05)$

${ }^{\S}$ Significantly lower than the 3 -month value $(\mathrm{p}<0.0001)$.
} 
Figure 1

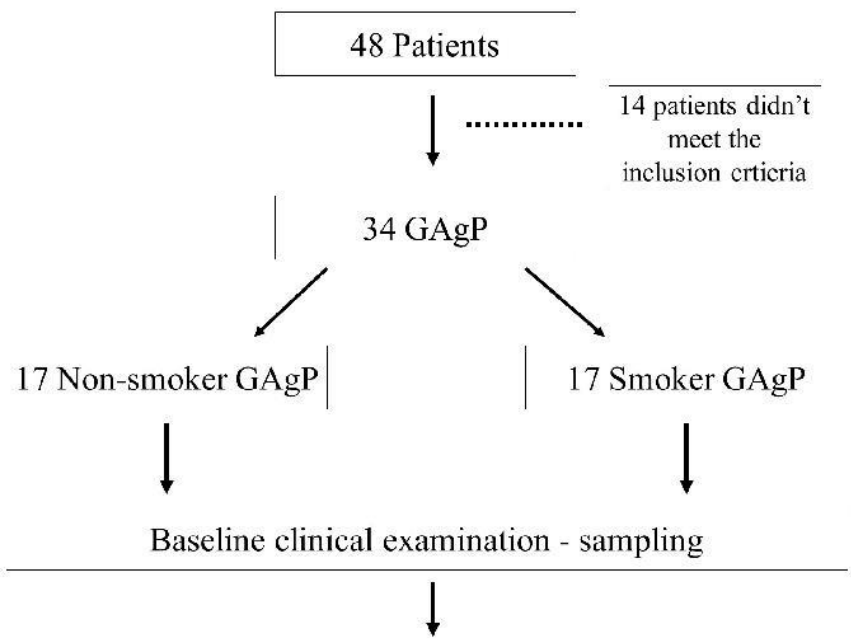

Non-surgical periodontal treatment

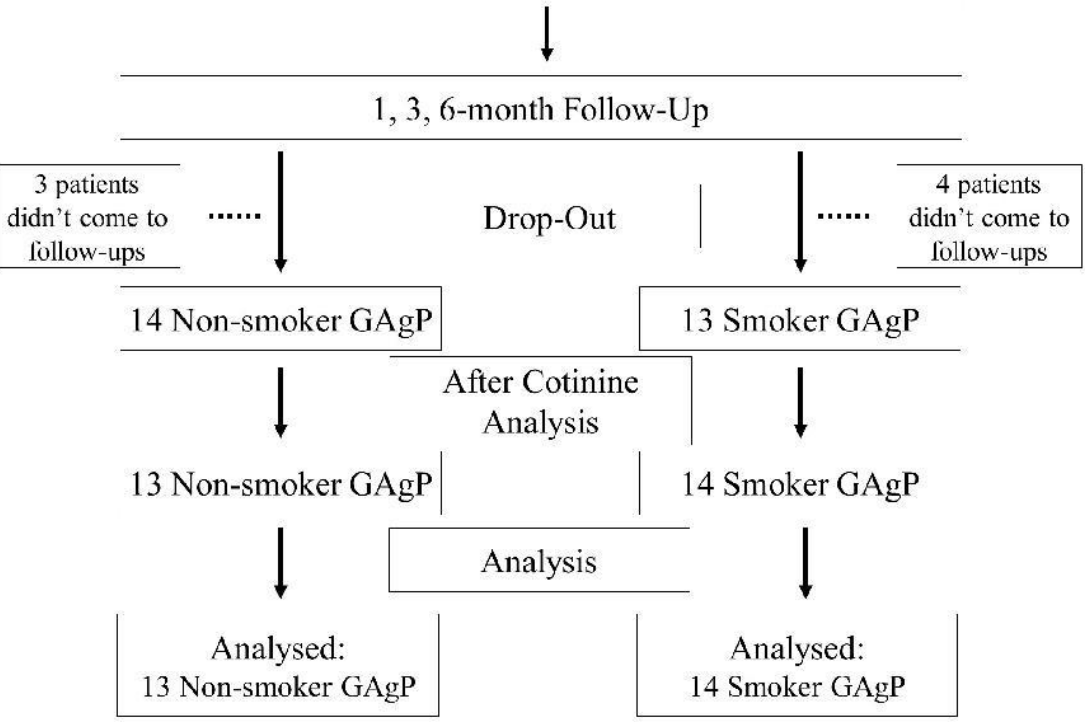


Figure 2
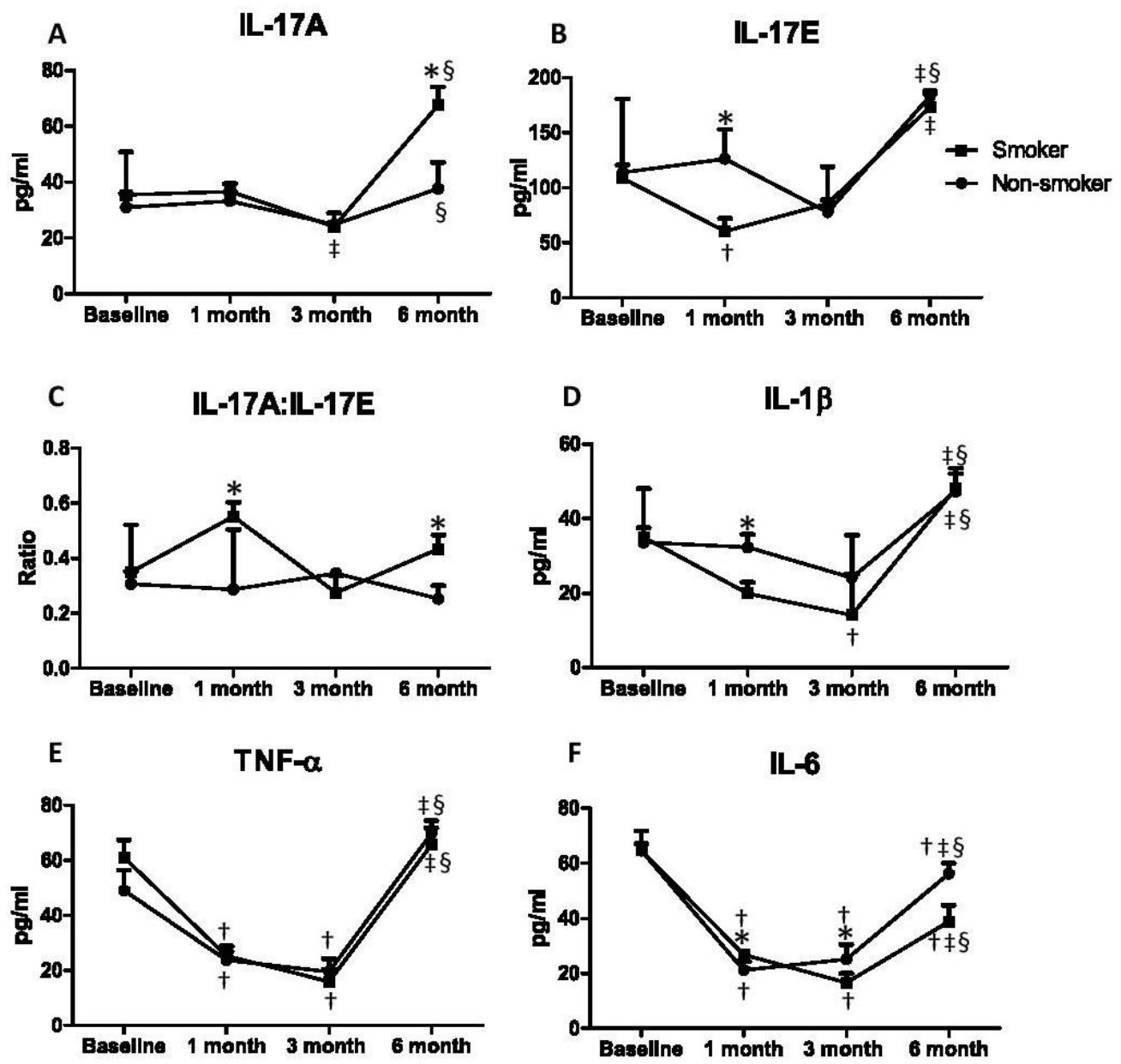
Figure 3
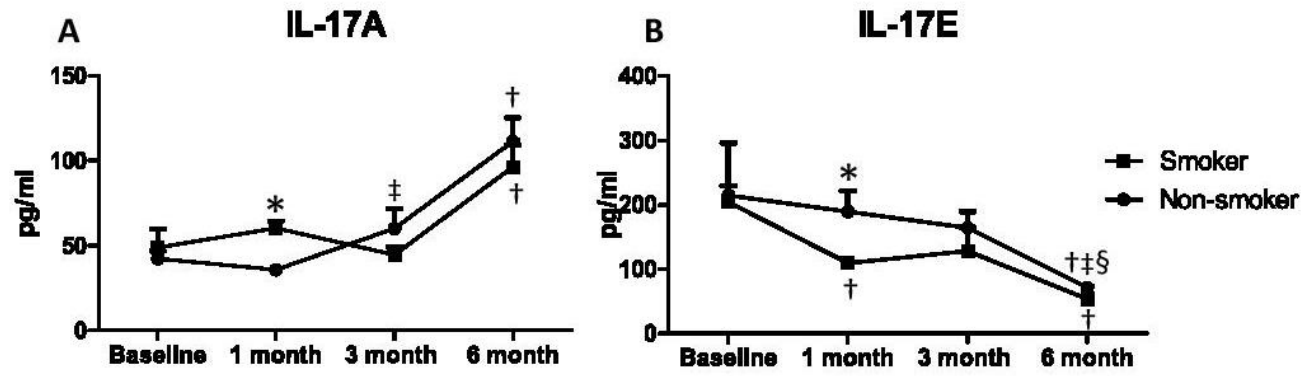

C

|L-17A:IL-17E

D

IL-1 $\beta$
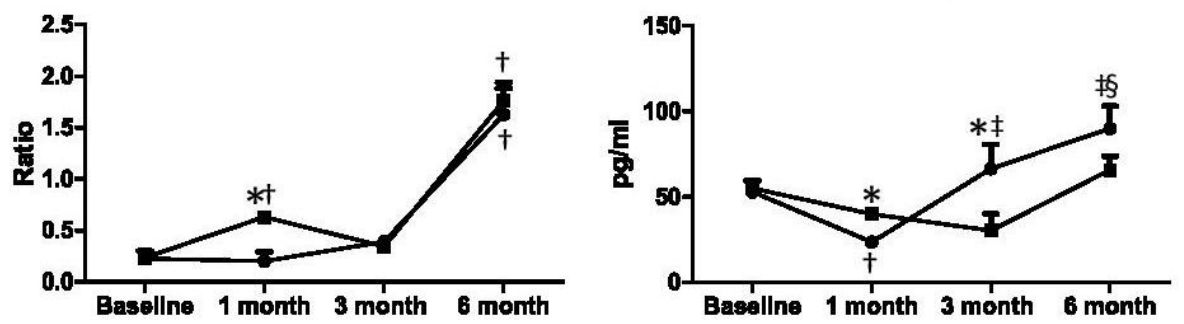

E TNF- $\alpha$
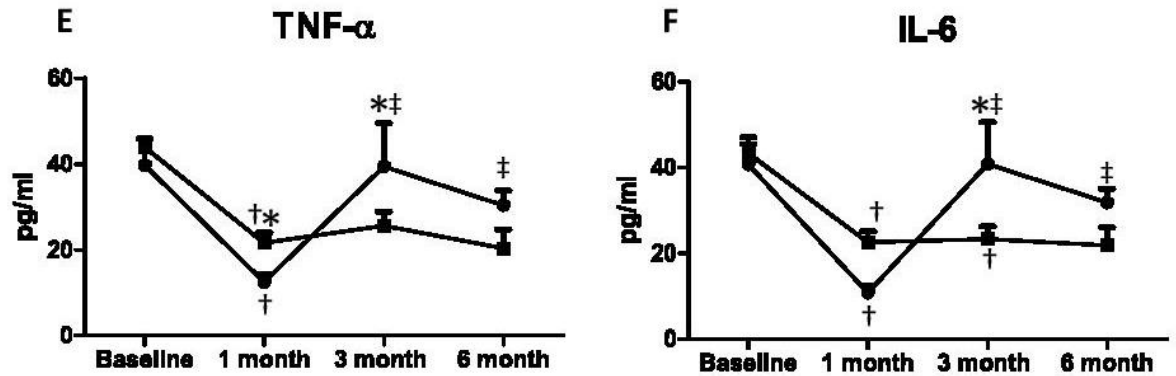
Figure 4
A A. actinomycetemcomitans
B
F. nucleatum
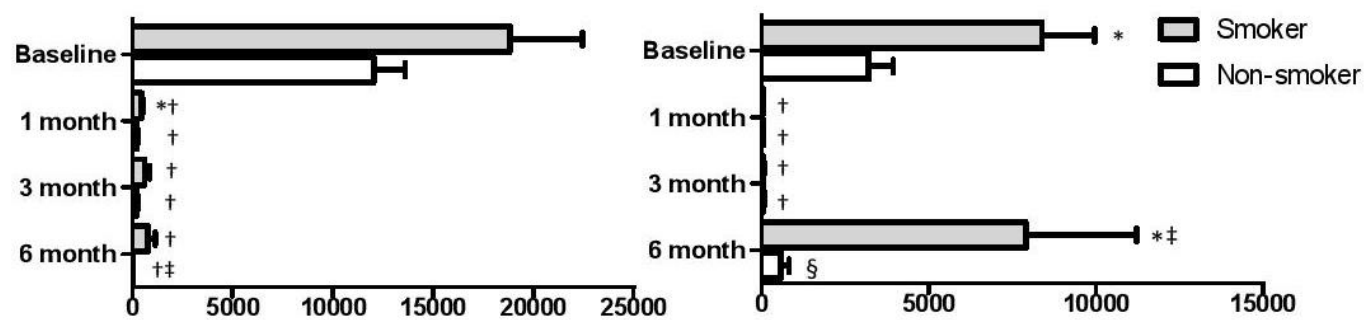

C

$P$. gingivalis

D

$P$. intermedia
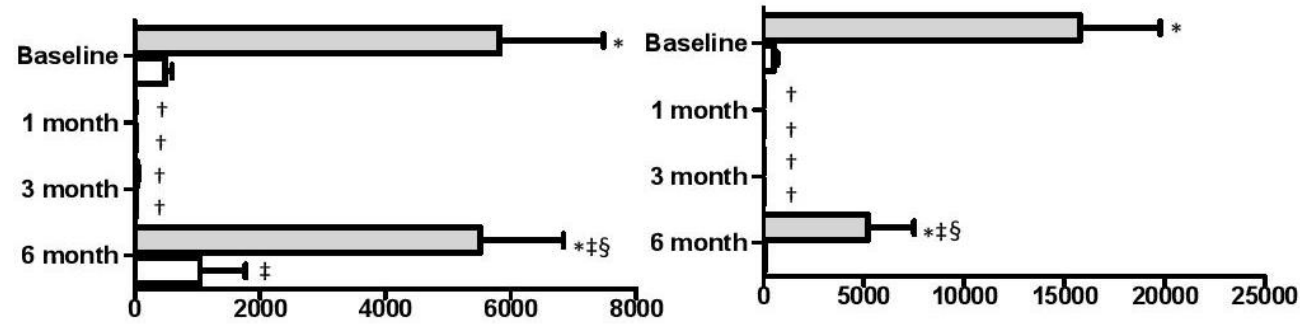

E

$P$. oralis

$\mathrm{F}$

T. denticola
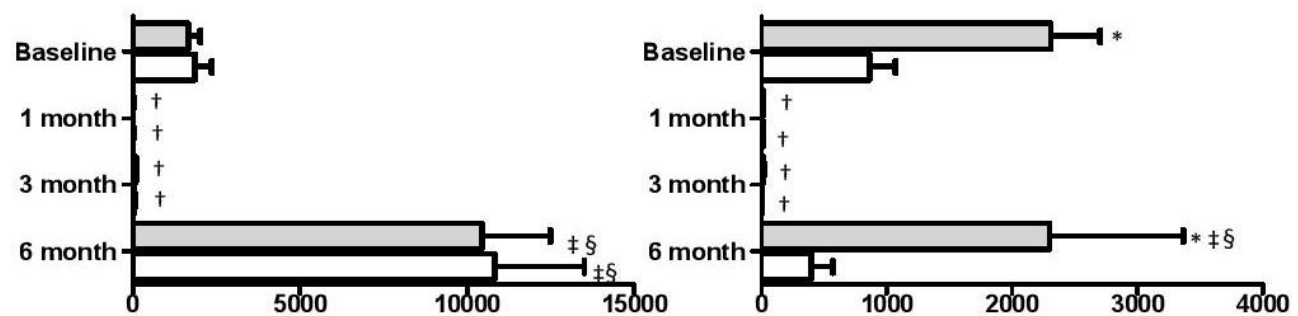

G

T. forsythia

$\mathrm{H}$

V. dispar
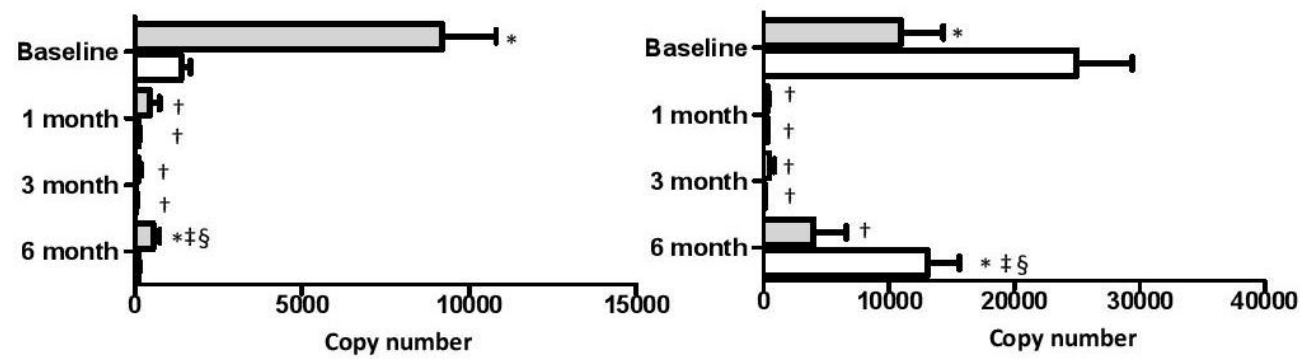
Figure 5
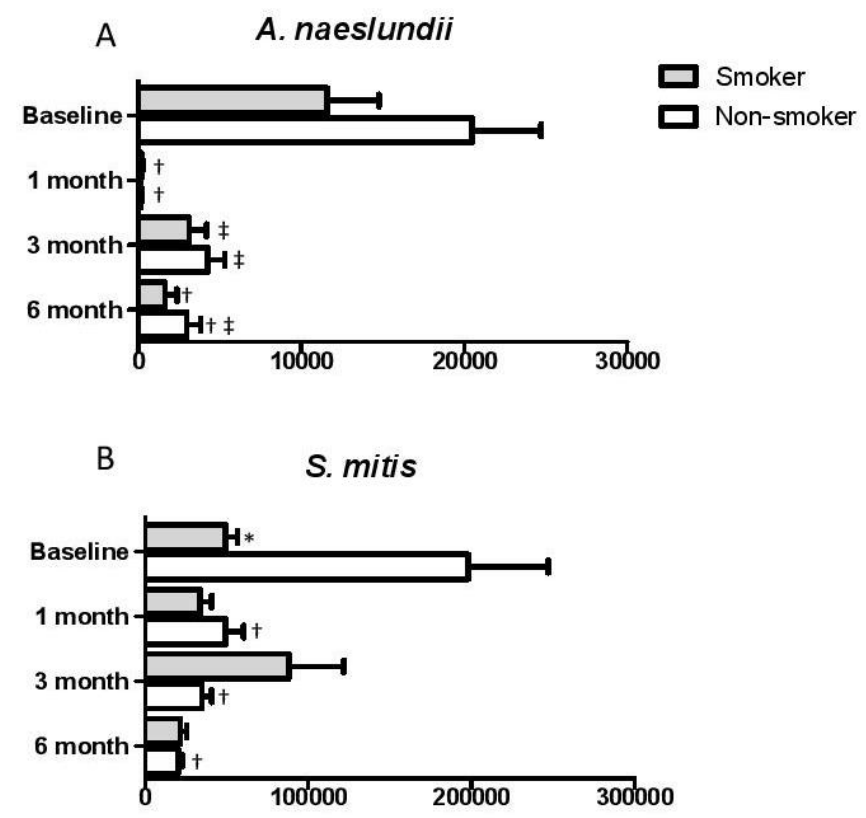

C S. oralis

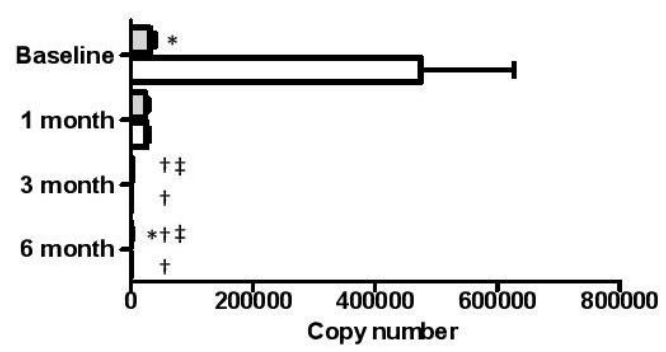

